\title{
La interactividad en las sedes webs corporativas: retos y oportunidades para las pymes
}

María García

\begin{abstract}
Phd. in Communication (University of Extremadura, Spain). She has taught in the department of Information and Communication at the University of Extremadura (Spain) from 2007 to 2011. She also does researches in the department Visiting Professor at several universities such as University of Brighton (UK), University of Nantes (France), Universidad Nova de Lisboa (Portugal) and Universidad Técnica Particular de Loja (Ecuador)
\end{abstract}

Ana Castillo Díaz

Lecturer at the Department of Information and Communication, University of Extremadura, Spain

M. Victoria Carrillo Durán

Visiting Professor at several Universities in Europe and America such as Turku University. Finland, University of Portsmouth. UK, University of Illinois. Chicago, University of Nantes. France, University of Canes. France, University of Salerno. Italy, University of Parma. Italy, Univerity of Bourgogne. France, Lodz Academy. Poland. Fellow of the European Advertising Academy and Iberoamerican Academy of Management.

La aplicación de las herramientas de Internet, y la interactividad que propician, ha modificado la forma de relacionarse de las empresas con sus públicos. Las sedes webs corporativas, como principal herramienta de comunicación planificada, deberían adaptarse a los requerimientos del medio y favorecer la interacción entre los usuarios y la organización. El presente trabajo analiza, mediante un análisis de contenidos y un análisis factorial, la predisposición de las Pequeñas y Medianas Empresas (PYMES) a la interacción con sus públicos en sus sedes webs y ofrece una panorámica de la situación actual. Los resultados obtenidos ofrecen una visión poco alentadora sobre la importancia que conceden a la interacción con sus públicos de interés a través de sus sedes webs corporativas ya que, aunque existe preocupación por 
garantizar la participación, no hay interés en fomentar la comunidad.

Palabras clave: Internet; Interactividad; PYMES.

The application of Internet tools, especially interactivity, has changed the way companies interact with their stakeholders. This paper analyzes, using a content analysis and a factorial analysis, small and medium-sized enterprise (SMEs) predisposition to interact with their audiences on their corporate websites, the main tool to planned communication, and provides an overview of the current situation. The results offer a very bleak picture of the importance SMEs give to interactivity on their websites. Even if there is concern to ensure participation, no interest in fostering community can be demonstrated.

Keywords: Internet; Interactivity; SMES.

Recebido em 25.09.2012 Aceito em 10.12.2012

\section{Introducción}

La interactividad puede considerarse como una característica intrínseca al medio Internet (RODRÍGUEZ ARDURA, 2000; LÓPEZ GARCÍA, 2005; MARTÍ PARREÑO, 2009), erigiéndose como uno de los pilares sobre los que se sustentan las estrategias de comunicación empresarial en la World Wide Web. A pesar de seguir siendo un asunto de actualidad en el mundo del marketing (VAN NOORT et al., 2012), existe un gran desconocimiento sobre la predisposición de las empresas a interactuar con sus públicos de interés en Internet.

Por otra parte, dentro del mundo de las organizaciones, las PYMES son empresas con entidad propia, diferenciadas de las grandes compañías cuyas características intrínsecas les hacen demandar estrategias a su medida. Aunque cada día más estudios abordan las particularidades de las PYMES, (BERTHON et al., 2008; MITCHELL et al., 2010) la implantación de Internet ha abierto una nueva brecha entre grandes empresas y las pequeñas y medianas para quienes la interactividad supone una gran oportunidad para crear comunidades (SADOWSKI et al., 2002).

Este estudio tiene por objeto estudiar el desarrollo que las PYMES hacen de la interactividad a través de sus portales webs corporativos desde la perspectiva del managment. Para ello se han seleccionado estadísticamente 231 sedes webs de PYMES ubicadas en la región española de Extremadura en las que se ha realizado un análisis de contenidos y un análisis factorial. 


\section{Las PYMES y las sedes webs corporativas}

Según el Directorio Central de Empresas (INE, 2012), a 1 de enero del año 2011 había en España 3.243.185 PYMES, es decir, empresas que emplean a menos de 250 trabajadores, tienen una facturación inferior a 50 millones de euros y/o un balance anual inferior a 43 millones de euros (EUROPEAN COMMISSION, 2006).

En un contexto globalizado, cambiante, fugaz e hiperconectado las PYMES disponen de un excelente instrumento mediante la cual comunicarse y transmitir su marca a los públicos: Internet. La Web es una amalgama de contenidos e informaciones donde la empresa se puede comunicar con sus públicos mediante distintas herramientas como las redes sociales, la publicidad digital, etc. Sin embargo, son los portales corporativos el canal controlado (BECERRA MUÑOZ, 2010), mediante el cual las organizaciones pueden comunicarse de forma directa con sus stakeholders. Por su parte los stakeholders conciben los websites como el canal a través del cual las organizaciones pueden ser vistas y entendidas (KENT et al., 2003).

La importancia que las PYMES conceden a Internet ha llevado a la proliferación, lenta pero constante, de empresas que disponen de sede web corporativa, tal y como demuestra el grafico 1 (véase al final del artículo). En el mismo se observa el porcentaje de PYMES con conexión a Internet y sitio web corporativo en España y su evolución en los últimos años.

Para este estudio, la muestra fue seleccionada entre PYMES ubicadas en la comunidad autónoma de Extremadura donde el 99,96\% de las empresas son PYMES, sumando así 65.463 en total (INE, 2012). En la comunidad extremeña se observa el mismo panorama que en el resto de España con una tendencia alcista en lo que a PYMES que disponen de sedes webs se refiere así como en el uso de las nuevas tecnologías tal y como demuestra la Encuesta de uso de TIC y comercio electrónico en las empresas (INE, 2012).

\subsection{Interactividad y PYMES}

La interactividad es un concepto multidisciplinar ampliamente tratado (JIANG et al., 2010). En el entorno online, la interactividad se entiende como la predisposición de la empresa a "escuchar" a sus públicos, bien sea de manera inmediata (interactividad de navegación) como a posteriori mediante el análisis y la adaptación del mensaje a las necesidades de los usuarios.

A pesar de la dificultad para establecer una definición válida desde el ámbito de la comunicación de masas, una propuesta que abarca el sentido más tridimensional de la interactividad sería la de Liu y Shrum (2002): el grado en que dos o más usuarios pueden actuar entre sí, mientras se comunican por medio de mensajes a través de un medio, y el grado en que estas relaciones están sincronizadas. 
Para Ind y Riondino (2001, p. 13) la interactividad es una de las principales características del medio en su relación con los públicos de interés ya que facilita:

a) el feedback, que a su vez tiene una doble dimensión, ya que contribuye tanto a comprobar la eficacia de la estrategia como a mejorar la orientación al cliente;

b) el diálogo con los usuarios: lo que ayuda a establecer relaciones estables y duraderas.

Disponer de sedes webs interactivas permitiría a las PYMES crear comunidades con clientes o proveedores (SADOWSKI et al., 2002) cuya lealtad será determinante para establecer relaciones a largo plazo. Esta necesidad de comunicación a través de las sedes webs viene marcada por el cambio en las relaciones que se establecen entre las empresas y los públicos, habiendo pasado de hablar de audiencias a hablar de comunidades (BIC GALICIA, 2010).

\section{Estudio exploratorio}

Con el objetivo de entender la predisposición de las PYMES hacia el uso de la interactividad en sus sedes webs corporativas, entendida como la actitud de escucha activa de las PYMES a las demandas de los nuevos usuarios de la Red, se ha llevado a cabo una revisión de 231 sedes webs corporativas. La muestra procede de la base de datos $\mathrm{SABI}^{1}$ que, en el mes de julio de 2011, incluía 465 PYMES ubicadas en la región de Extremadura y con sede web activa en el año 2009. De ellas, aplicando un error máximo de un $2 \%$ con un nivel de confianza del $95 \%$, se seleccionaron aleatoriamente 231.

Para conseguir este objetivo se ha realizado un análisis dividido en dos pasos.

\subsection{Análisis de contenidos}

En primer lugar, para estudiar los mensajes expuestos en las sedes webs corporativas de las PYMES se ha optado por un análisis de contenido como técnica que se centra en "la descripción objetiva, sistemática y cuantitativa del contenido manifiesto de la comunicación" (BERELSON, 1952). Es decir, mediante el análisis de contenidos se analizaran fragmentos de texto segmentado en unidades que posteriormente serán codificadas. Estos fragmentos de texto no son entes aislados, sino que

\footnotetext{
${ }^{1}$ SABI son las siglas de la base de datos Sistema de Análisis de Balances Ibéricas. Es una base de datos financiera que incluye las principales empresas españolas y portuguesas. Tiene registradas más de 320.000 empresas portuguesas y 550.000 empresas españolas.
} 
forman parte de un modelo propuesto fruto de una exhaustiva revisión bibliográfica (Tabla 1, véase al final del artículo).

El modelo se estructura alrededor a diversas categorías o variables que se agrupan y se relacionan de forma más o menos directa y cuya gestión conjunta determinará la predisposición hacia la correcta aplicación de la interactividad en las sedes webs. De esta forma, los resultados obtenidos permiten conocer la situación de las PYMES extremeñas en relación con la variable interactividad.

De acuerdo con lo expuesto en la Tabla 1 (véase al final del artículo), la variable independiente del estudio es la interactividad. Como se ha expuesto, se pretende conocer la predisposición hacia la interacción de las PYMES con sus públicos en las sedes webs corporativas. Esta variable independiente se configura mediante la suma positiva de los factores (o variables dependientes), categorías e indicadores en la configuran.

De la variable independiente (interactividad) se desprenden una serie de variables dependientes (o factores) que son la interacción permanente, la personalización y el $\mathrm{CRM}^{2}$ (Tabla 1 , véase al final del artículo). Se puede decir que para que una sede web sea considerada interactiva, debe incluir herramientas de interacción permanente, personalizar las comunicaciones y mostrar interés por la gestión de las relaciones con los clientes.

A su vez, estos factores se concretan en categorías que serán las unidades de contexto (ANDREÚ, 2000) que deberán ser examinadas para poder caracterizar y codificar los indicadores. Es decir, las categorías aportarán la información necesaria del contexto para el análisis de los fragmentos de texto o indicadores.

Los indicadores se han adscrito a una categoría (Tabla 1, véase al final del artículo) según la revisión bibliográfica realizada, pero es importante no encasillarlos ni hacerlos excluyentes puesto que la suma positiva de todos estos indicadores dará lugar a una predisposición favorable hacia la interactividad. Se han dividido para su mejor análisis, pero es importante remarcar que se complementan y están interconectadas, tal y como se comprobará posteriormente en el análisis factorial.

\subsection{Análisis factorial}

Este modelo y las categorías formuladas han sido ya validados desde el punto de vista teórico (tabla 1, véase al final del artículo) pero es necesario comprobar si se ajustan o no a la realidad analizada, saber si las PYMES asocian esos indicadores a las categorías presentadas y si se relacionan entre ellos de la misma forma que en el modelo propuesto. Para ello se ha realizado un análisis factorial que se puede definir como "un método multivariante que intenta explicar un conjunto de variables

${ }^{2}$ Customer Management Relationship voz inglesa que hace referencia a la gestión de las relaciones con los clientes. 
observables mediante un número reducido de variables no observables llamadas factores" (LEÓN DUARTE, 2005, p. 56).

El análisis factorial de los resultados obtenidos en el análisis de contenidos permite comprobar las posibles modificaciones del modelo teórico propuesto según las acciones que realizan las PYMES en sus websites.

Dicho análisis factorial por componentes principales sintetiza la información de los datos substituyendo las variables originales por los nuevos factores, de manera que, si se considera un número menor de estas últimas, expliquen una porción suficientemente grande de varianza inicial. Estas variables nuevas se llaman componentes principales 0 factores y no contienen información redundante.

Las categorías propuestas en el modelo pasarán ahora a denominarse factores, ya que como el nombre del método indica, han sido "factorizadas" (grafico 2, véase al final del artículo). Los factores obtenidos explicarán la realidad estudiada, si bien será necesario renombrarlos, puesto que lo que se obtiene tras la aplicación del análisis factorial es un conjunto (o factores) que agrupa un número determinado de variables pero cuyo nombre podrá variar en función del nuevo reparto

\section{Resultados}

Para ser coherentes con el planteamiento expuesto, se van a explicar los resultados de ambos análisis por separado y posteriormente se unirán mediante la explicación de las conclusiones.

\subsection{Resultados del análisis de contenidos}

Para entender correctamente los resultados obtenidos en el análisis factorial es necesario hacer referencia a las puntuaciones obtenidas en cada indicador. En el Grafico 3 (véase al final del artículo) se observan las medias alcanzadas en cada indicador. Teniendo en cuenta que todos los indicadores podían alcanzar una puntuación máxima de 4 puntos, las medias obtenidas permiten avanzar la escasa atención que prestan las PYMES a determinados aspectos de la interactividad.

En la categoría de "interacción permanente", el indicador al que más atención parecen prestarle las PYMES es al de participación. En la misma categoría, hay que destacar los bajos resultados logrados en el indicador "fomento de la comunidad". Ya se ha apuntado a que la creación de comunidades con clientes o proveedores es especialmente pertinente en el caso de las PYMES. Los valores obtenidos se agrupan alrededor de la puntuación más baja, con una media de 1,09.

En la categoría de "personalización", el indicador "distingue públicos" muestra una clara orientación de las PYMES a los clientes, ignorando así otros tipos de públicos de interés. A pesar de la clasificación tomada como referencia (ver Tabla 1 al final del artículo) se entiende como una gradación de los públicos en función de su importancia para las empresas, 
un $11,3 \%$ de las PYMES se dirigen en sus sede web al publico impactado frente al 3\% que toma en consideración al publico relevante.

La última categoría estudiada (CRM), arroja resultados que validan lo observado en el resto de categorías. Tanto los resultados de la categoría interacción permanente como los obtenidos en el CRM ponen de manifiesto que la inmediatez en las relaciones, típicas de las aplicaciones 2.0 (como los chats o los foros de discusión), no son una prioridad para las PYMES.

\subsection{Resultados del análisis factorial}

Una vez analizados los indicadores propuestos en el modelo, se validó su agrupación mediante el análisis factorial. La pertinencia del análisis factorial en la investigación quedó demostrada por el determinante de la matriz de correlaciones, el test de esfericidad de Barlett, el índice KMO de Kaiser-Meyer-Olkin, la medida de adecuación de la muestra (MSA) y la matriz de correlaciones anti-imagen (GARCÍA JIMÉNEZ et al., 2000; HAIR et al., 1999) que arrojaron resultados que permitían la realización del análisis.

Partiendo de la base teórica que sustenta la inclusión de todos los indicadores tenidos en cuenta, se podrá adjudicar un nombre a cada factor obtenido, ya que los factores extraídos del análisis factorial no necesariamente tienen que coincidir con los propuestos en la parte teórica. Los nuevos factores se compondrán de diversos indicadores que determinarán el nombre de la agrupación final.

Los resultados obtenidos arrojan algunos cambios en el modelo propuesto: el factor 1 equivaldría $100 \%$ con la variable interacción permanente, pero los factores 2 y 3 han sufrido algunos cambios. El factor 2 estaría compuesto por las variables FAQS y distingue públicos mientras que el factor 3 se configuraría mediante la variable contacto.

A continuación se explica la configuración de cada factor y su configuración en función de los resultados obtenidos en el análisis factorial.

factor 1. Interacción permanente: Las variables asignadas al primer factor coinciden plenamente con las propuestas en el modelo teórico. Son la suscripción, el fomento de la comunidad, el archivo de noticias y la participación. Esta similitud tiene como consecuencia que el factor se haya denominado interacción permanente, ya que se ha configurado a base de variables que fomentan el contacto y la interacción de los públicos de interés con la organización. Este componente obtuvo un valor propio de 1,82 y explicó un $24,47 \%$ del total de la varianza tras la factorización de la variable interactividad;

factor 2. Segmentación y ayuda: Las variables incluidas en el segundo factor han sido cuestiones de diversa índole que en el modelo propuesto estaban agrupadas en categorías distintas. 
Las variables que configuran el factor 2 son las FAQs y distingue públicos.

Estas dos variables no tienen una correlación muy alta $(0,119)$ a pesar de lo cual las PYMES parecen dedicarles una atención similar. Tal y como se ha observado en el análisis univariable son dos cuestiones que reciben una puntuación media bastante escasa y que requieren de un análisis previo de los públicos de interés que las PYMES parecen no estar realizando. Esta escasa atención a las necesidades de los usuarios proviene de la identificación de los públicos de interés únicamente con los clientes. Fruto de esta interpretación es que se haya etiquetado este factor con el nombre de segmentación y ayuda. Este componente obtuvo un valor propio de 1,12 y explicó el $16,74 \%$ de la varianza;

factor 3. Contacto: Este factor ha quedado determinado con una única variable: el contacto. Esta variable es la más homogénea de todas las estudiadas, es muy compacta y tiene una baja correlación con el resto de variables.

La independencia de esta variable podría venir dada por la "necesidad" de incluir formas de contacto en las webs corporativas, lo cual no quiere decir que estas formas de contacto se integren en una estrategia de marca 2.0. Es decir, las PYMES entienden que el contacto con la empresa es obligatorio, de ahí la fortaleza y la independencia de la variable que, a diferencia de las otras, suele constituir una sección en sí misma que se entiende imprescindible, no discutible. El resto de aplicaciones o herramientas tienen un índice de aleatoriedad mayor.

\section{Conclusiones}

En lo que al factor 1 se refiere (interacción permanente) se puede decir que el escaso aprovechamiento que las PYMES hacen de la interactividad propia de Internet se da en un doble sentido: ausencia de interactividad tecnológica y ausencia de interacción con los públicos. Las PYMES no demuestra ninguna predisposición a la "comunicación interactiva con" (De Chernatony, 2001) los usuarios.

Las PYMES entienden que, en caso de entrar en contacto con los usuarios, este contacto debe ser iniciado por los públicos de interés. A pesar de esto carecen de mecanismos que permitan a los usuarios iniciar la conversación. El correo electrónico o un formulario de contacto son las principales herramientas detectadas.

Esta conclusión concuerda con lo expuesto por Moreno y Capriotti (2006) según los cuales las PYMES se encuentran en una fase muy inicial de desarrollo y aplicación de la interacción o del diálogo con sus públicos dentro de sus webs corporativas, ya que adoptan una postura principalmente unidireccional y expositiva. 
Respecto a sus stakeholders, para poder interactuar con sus públicos de interés las PYMES deberían conocer primero quiénes son estos públicos (CARRILLO DURÁN et al., 2005) de forma que la interacción vaya encaminada a conseguir unos objetivos comunicativos coherentes con la estrategia global de la empresa y con las necesidades de esos públicos de interés.

El indicador que se ha utilizado para medir la relación de los públicos y las PYMES arroja una conclusión clara: las PYMES se dirigen en sus webs corporativas únicamente a sus clientes.

Del factor 3 (contacto) se desprende una conclusión principal: las empresas están interesadas en ser contactadas en privado, en iniciar una conversación con cada usuario de forma individual, utilizando medios de comunicación tradicionales. Sin embargo, no parecen estar interesadas en ser ellas las que inicien el contacto tal y como se indicó en los resultados del factor 1 .

La tradición de los medios de comunicación de masas unidireccionales, pesa todavía mucho en las estrategias comunicativas de las PYMES. Así, se limitan a utilizar sus sedes webs como escaparates digitales en los que exponer aquellas informaciones que les interesan a ellas sin posibilidad de obtener un feedback $\mathrm{ni}$ inmediato $\mathrm{ni}$ en colaboración con el resto de visitantes.

Para solventar las deficiencias detectadas en esta investigación se hacen una serie de recomendaciones a las PYMES que puedan guiar el desarrollo de estrategias de comunicación a través de su sede web.

En primer lugar entender que la interactividad es una característica intrínseca al medio Internet sin la cual, ninguna acción de comunicación estará completamente desarrollada e integrada en el medio. Es de vital importancia que la interactividad no sea entendida como un complemento a la web, sino que forme parte del proceso de desarrollo de la misma.

En segundo lugar, la interactividad permitiría a las PYMES trasladar esa cercanía al consumidor (Berthon et al., 2008) mediante aplicaciones de CRM sencillas. Trasladar la cercanía y la familiaridad característica de las PYMES en el entorno offline al contexto online les permitirá diferenciarse de las grandes empresas.

Pero cualquier esfuerzo encaminado a maximizar el efecto de las acciones de comunicación en la sede web perderá eficacia si, previamente, las PYMES no realizan un trabajo de análisis e investigación de las necesidades y deseos de sus públicos de interés. Saber a quién van dirigidas las acciones de comunicación se antoja imprescindible.

\section{1 limitaciones y futuras lineas de investigación}

Los resultados obtenidos en esta investigación pueden estar condicionados por una serie de aspectos que la limitan a nivel conceptual y metodológico lo que representa, a su vez, unos nuevos futuros caminos de investigación. 
En primer lugar, la muestra se ciñe a la comunidad autónoma de Extremadura. Este escollo podría ser solventado ampliando el estudio a otras comunidades autónomas e incluso a otros países.

Además, el análisis de los determinantes de la interacción entre las PYMES y sus interlocutores online podría complementarse con el análisis de la predisposición a la interactividad de las empresas en el entorno offline.

Para continuar, el estudio se centra en el análisis de la interactividad que ofrecen las sedes webs corporativas como principal escaparate de la comunicación planificada online. Sin embargo, el auge y la importancia que están tomando en los últimos años otras plataformas de comunicación como las redes sociales o los blogs hacen recomendable el estudio de las estrategias de comunicación de las PYMES en este tipo de plataformas.

Basándose en los resultados obtenidos y en las limitaciones expuestas, futuras investigaciones podrían examinar la evolución de las sedes webs utilizando fuentes de datos y metodologías alternativas.

\section{Bibliografía}

ALONSO, J. Comunicar en el web: propuesta de criterios para analizar sitios en Internet. Teknokultura, v. 7, 2007. Disponible en: <http://teknokultura.uprrp.edu/pdf/alonso.pdf>. Consulta: 07/05/2011.

ÁLVAREZ RODRÍGUEZ, M. L et al. Reputación y responsabilidad desde las webs corporativas. Área abierta, n. 26, p. 1-22, 2010. Disponible en: $<$ http://revistas.ucm.es/index.php/ARAB/article/view/ARAB1010230001A >. Consulta: 09/05/2011.

ANDRÉU ABELA, J. Las técnicas de análisis de contenido: una revisión actualizada. Fundación Centro Estudios Andaluces, Universidad de Granada, v.10, n. 2, p. 1-34, 2000. Disponible en: <http://public.centrodeestudiosandaluces.es/pdfs/S200103.pdf>.

Consulta: 20/05/2009.

ANSARI, A. M.; MELA, C. F. E-customization. Journal of Marketing Research, v. 40, p. 131-145, 2003. Disponible en: $<$ http://faculty.fuqua.duke.edu/ mela/bio/papers/Ansari_Mela_2003.pdf $>$. Consulta: 10/04/2012.

BECERRA MUÑOZ, E. El escaparate online de la empresa. Revista Icono 14, n. 15, p. 207-219, 2010. Disponible en: $<$ http://www.icono14.net/revista/num15/14_icono15_elenabecerra.pdf $>$. Consulta 06/10/2011.

BERELSON, B. Content analysis in comunication research. Free Press: Glencoe, 1952.

BERTHON, P.; EWING, M. T.; NAPOLI, J. Brand management in small to medium-sized enterprises. Journal of Small Business Management, v. 46, n. 1 , p. 27-45, 2008. 
BIC GALICIA. Manuales prácticos de la pyme: como crear una marca. $2010 . \quad$ Disponible en: < http://www.bicgalicia.es/dotnetbic/Portals/0/banner/ARCHIVOS/Manuales\%20Pymes/13 MARCA_Cast.pdf>. Consulta: 05/05/2011.

CARRILLO DURÁN, M. V. La interactividad: un reto para la publicidad en el entorno digital online. Revista Zer. Revista de Estudios de Comunicación, v. 10, p. 9-24, 2005. Disponible en: $<$ http://www.ehu.es/zer/hemeroteca/pdfs/zer18-01-carrillo.pdf $>$. Consulta 30/11/2009.

CASTILLO DÍAZ, A. La comunicación de las marcas universitarias españolas a través de sus sedes webs, 2007. Tesis (Doctoral) Universidad de Extremadura, 2007.

DE CHERNATONY, L. Succeeding with brands on the Internet. Journal of Brand Management, v. 8, n.3, 2001. Disponible en: $<$ http://www.brandchannel.com/images/papers/success.pdf $>$. Consulta: 30/12/2009.

DÍAZ SÁNCHEZ, E. et al. Evaluación de la eficacia de las páginas web: un análisis de contenido de las principales compañías aéreas. SILVA, E. J. de C.; CASTRO, J. D. de (Coord.). Universidad, Sociedad y Mercados Globales. 2008. p. 279-293. Disponible en: $<$ http://dialnet.unirioja.es/servlet/articulo?codigo=2751745> $>$. Consulta: 09/10/2010.

DOU, W.; KRISHNAMURTHY, S. Using brand web sites to uild brands online: a product vs. service brand comparison. Journal of Advertising Research, n. 47, v. 2, p. 193-206, 2007. Disponible en: $<$ http://fbstaff.cityu.edu.hk/mkwydou/Using\%20Brand\%20Websites\%20to\%20Build\%20Bra nds\%20Online_A\%20Product\%20versus\%20Service\%20Brand\%20Comparison.pdf $>$.

Consulta: $13 / 06 / 2012$.

EUROPEAN COMMISION. The new SME definition. 2006. Disonible en: $<$ http://ec.europa.eu/enterprise/policies/sme/files/sme_definition/sme_user_guide_en.pdf $>$.C onsulta: 20/01/2011.

GARCÍA BORBOLLA, A. et al. Empirical evidence concerning smes' corporate websites: explaining factors, strategies and reporting. The International Journal of Digital Accounting Research, v. 5, n. 10, p. 171202, 2005.

GARCÍA JIMÉNEZ, E. et al. Análisis factorial. Madrid: La Muralla, 2000.

GÓMEZ, L. M.; CHALMETA, R. Corporate responsibility in U.S. corporate websites: a pilot study en Public Relations Review, n. 37, p. 93-95, 2011.

HAIR, J.F. et al. Análisis multivariante. Madrid: Prentice Hall, 1999.

HUERTAS ROIG, A.; XIFRA TRIADÚ, J. ¿Marcas o genéricos? La comunicación en línea de las marcas farmacéuticas. Revista Zer. Revista de Estudios de Comunicación, v. 14, n. 27, p. 251-270, 2009. Disponible en: <http://www.ehu.es/zer/zer27/zer27-17-huertas.pdf>. Consulta: 20/06/2011.

IND, N.; RIONDINO, M. Branding on the web: a real revolution? Brand Management, v. 9, n.1, p. 8-19, 2001. 
JIANG, Z. et al. Effects of interactivity on website involvement and purchase intention. Journal of the Association for Information Systems, v. 11, n.1, p. 1-47, 2010. Disponible en: $<$ http://www.comp.nus.edu.sg/ jiang/Jiang\%20et\%20al.\%202009.pdf $>$. Consulta: 02/06/2011.

KENT, M. L.; TAYLOR, M.; WHITE, W. J. The relationship between Web site design and organizational responsiveness to stakeholders. Public Relations Review, v. 29, n. 1, p. 63-77, 2003. Disponible en: <http://facultystaff.ou.edu/K/Michael.L.Kent-1/PDFs/KentTaylorWhite.pdf>. Consulta: 20/06/2008.

LEÓN DUARTE, J. A. Metodología para la detección de requerimientos subjetivos en el diseño de producto. Tesis (Doctoral) - Universidad Politécnica de Cataluña, 2005. Disponible en: <http://tdx.cat/handle/10803/6840>. Consulta: 06/06/2011.

LIU, Y.; SHRUM, L. J. What is interactivity and is it always such influence of interactivity on advertising effectiveness. Journal of Advertising, v. 31, n. 4, p. 53-64, 2002.

LÓPEZ GARCÍA, G. Modelos de comunicación en Internet. Valencia: Ed. Tirant lo Blanch, 2005.

MACIAS, W. A Beginning Look at the effects of interactivity, product involvement and Web experience on comprehension: brandWeb sites as interactive advertising. Journal of Current Issues \& Research in Advertising, v. 25, n. 2, p. 31, 2003.

MARTÍ PARREÑO, J. Marketing y publicidad en Internet. Paracuellos de Jarama: StarBook, 2009.

MARTÍ PELLÓN, D.; ÁLVAREZ RODRÍGUEZ, M. L.; DOMÍNGUEZ QUINTAS, S. Imagen corporativa web: análisis del discurso de empresas de internet. Revista electrónica Razón y Palabra, n. 69, 2009. Disponible en: $<$ http://www.razonypalabra.org.mx/comEstrategica/JUL30.html $>$.

Consulta: 03/07/2010.

MITCHELL, R; HUTCHINSON, K.; BISHOP, S. Interpretation of the retail brand: an SME perspective. International Journal of Retail and Distribution Management, v. 40, n. 2. p. 157-175, 2011.

MORENO, A.; CAPRIOTTI, P. La comunicación de las empresas españolas en sus webs corporativas: análisis de la información de responsabilidad social, ciudadanía corporativa y desarrollo sostenible. Revista Zer. Revista de Estudios de Comunicación, n. 21, p. 47-62, 2006. Disponible en: <http://www.ehu.es/zer/hemeroteca/pdfs/zer21-04-moreno.pdf>. Consulta: 13/06/2008.

RODRÍGUEZ ARDURA, I. Marketing. com. Madrid: Esic Editorial, 2000.

SADOWSKI, B. M.; MAITLAND, C.; VAN DONGENC, J. Strategic use of the Internet by small and medium-sized companies: an exploratory study. Information Economics and Policy, v. 14, n.1, p. 75-93, 2002. 
VAN NOORT, G. et al. Interactivity in brand Web sites: cognitive, affective, and behavioral responses explained by consumers' online flow experience. Journal of Interactive Marketing, v. 26, n. 4, p. 223-234, 2012.

\section{Otros recursos}

Disponible en: < $\underline{w w w . i n e . e s}>$. Consulta: 13/06/2008.

\section{Anexos}

Gráfico 1 - Porcentaje de PYMES con conexión a Internet y sitio web corporativo en España y su evolución en los últimos años

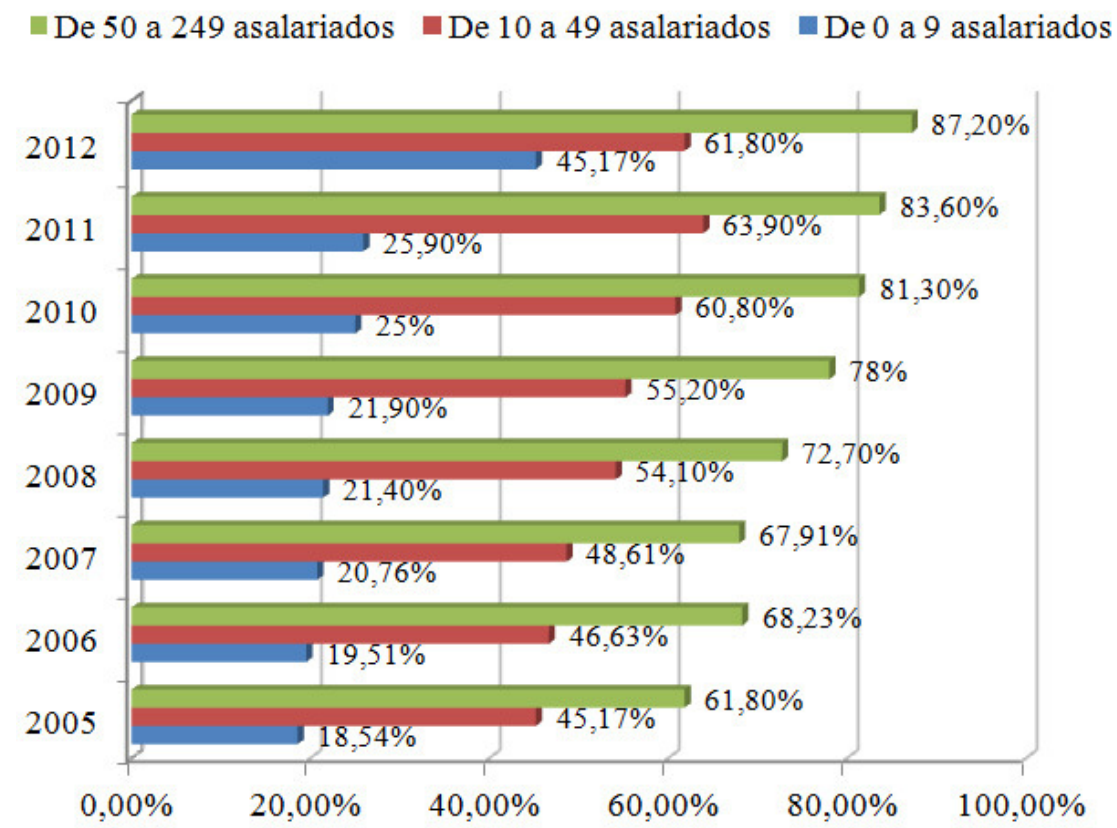

Fuente: Elaboración propia a partir de los datos del INE (2012).

Datos a 1 de enero de ese año.

Grafico 2 - Paso del análisis de contenidos al análisis factorial

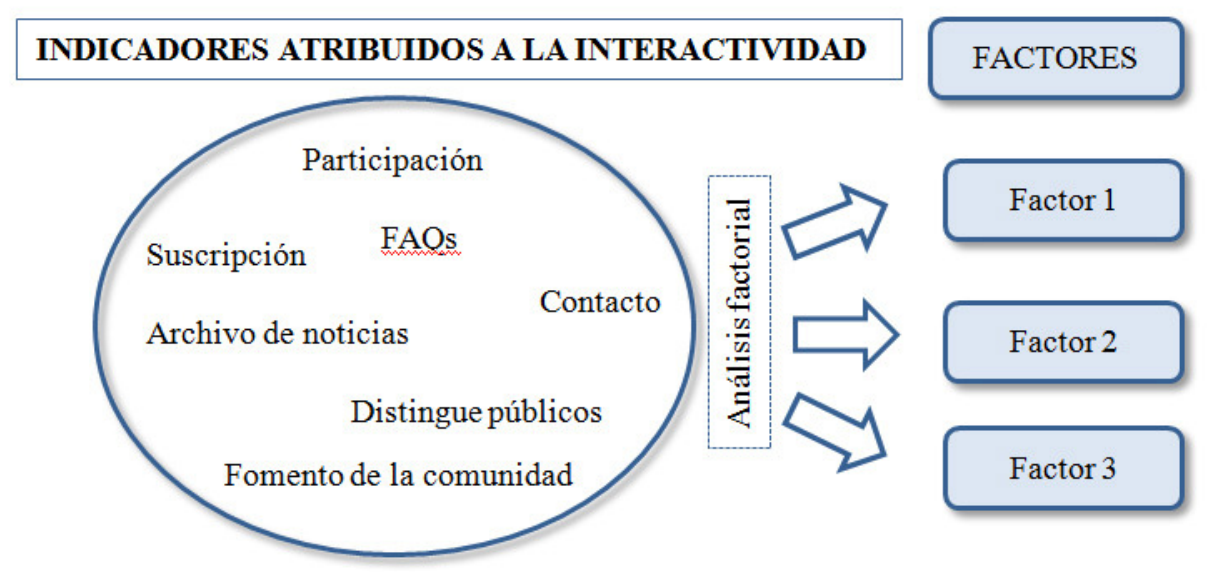

Fuente: Elaboración propia.

Grafico 3 - Medias obtenidas en los indicadores 


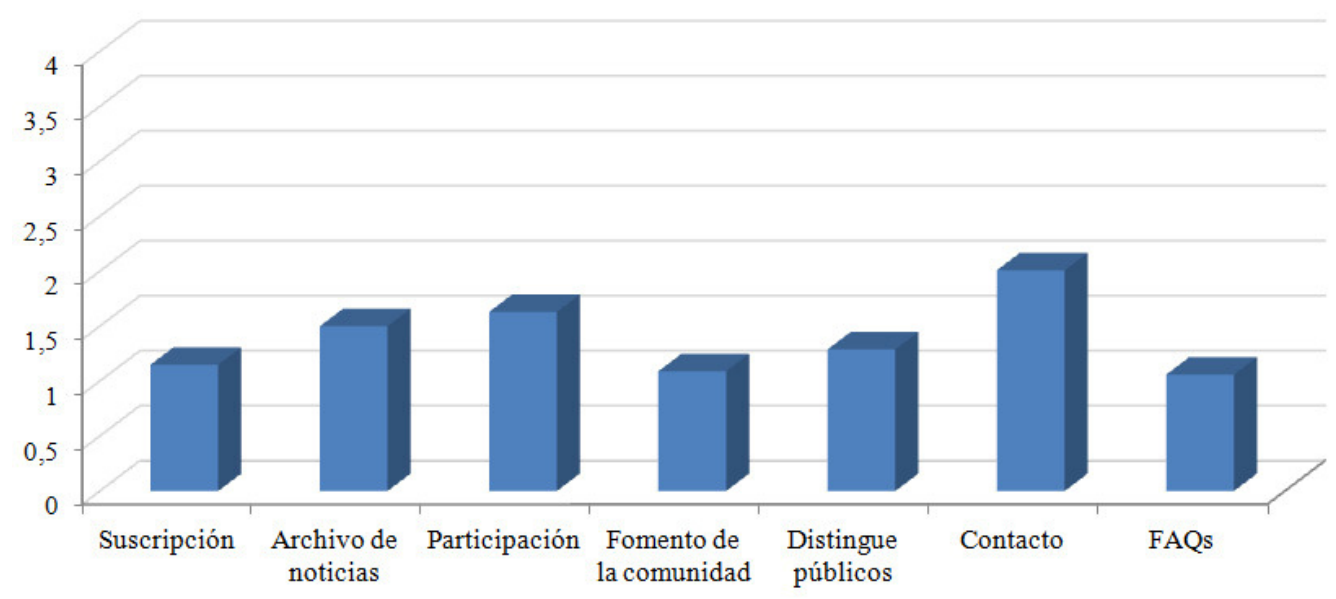

Fuente: Elaboración propia.

Tabla 1 - Modelo ideal propuesto y principales autores que utilizan los indicadores propuestos

\begin{tabular}{|c|c|c|c|c|}
\hline Factores & Categorías & Indicadores & Puntuación & $\begin{array}{l}\text { Autores que lo han } \\
\text { utilizado }\end{array}$ \\
\hline \multirow{16}{*}{ 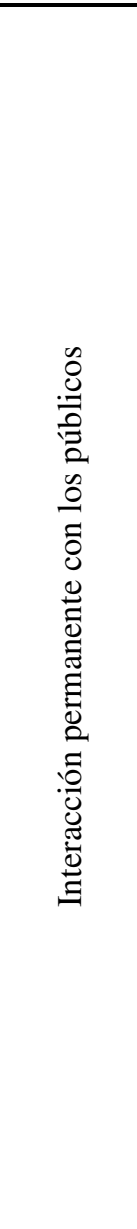 } & \multirow[t]{4}{*}{ Suscripción } & Ambas aplicaciones (RSS y Boletines) & 4 & \multirow{4}{*}{$\begin{array}{r}\text { Huertas Roig y Xifrá } \\
\text { Triadú (2009); Gómez y } \\
\text { Chalmeta (2011) }\end{array}$} \\
\hline & & RSS & 3 & \\
\hline & & Newsletter/boletin & 2 & \\
\hline & & No dispone & 1 & \\
\hline & \multirow[t]{4}{*}{ Archivo de noticias } & Todas las informaciones & 4 & \multirow{4}{*}{$\begin{array}{r}\text { Alonso (2007); Gómez y } \\
\text { Chalmeta (2011) }\end{array}$} \\
\hline & & Noticias propias & 3 & \\
\hline & & Recoge informaciones de los medios & 2 & \\
\hline & & No dispone & 1 & \\
\hline & \multirow[t]{4}{*}{ Participación } & Ambas herramientas & 4 & \multirow{4}{*}{$\begin{array}{r}\text { Dou y Krishnamurthy } \\
\text { (2007); Martí Pellón et al } \\
\text { (2009); Huertas Roig y } \\
\text { Xifrá Triadú (2009) } \\
\text { Álvarez Rodríguez et al } \\
\text { (2010); Gómez y } \\
\text { Chalmeta (2011) }\end{array}$} \\
\hline & & Comentarios públicos & 3 & \\
\hline & & $\begin{array}{l}\text { Comentarios mediante } \\
\text { formularios/encuestas }\end{array}$ & 2 & \\
\hline & & No permite & 1 & \\
\hline & \multirow{4}{*}{$\begin{array}{l}\text { Fomento de la } \\
\text { comunidad }\end{array}$} & Ambas herramientas & 4 & \multirow{4}{*}{ Anasri y Mela (2003) } \\
\hline & & Programas de fidelización & 3 & \\
\hline & & $\begin{array}{l}\text { Enlace a redes sociales/blogs o } \\
\text { comentarios de otros usuarios }\end{array}$ & 2 & \\
\hline & & No dispone & 1 & \\
\hline 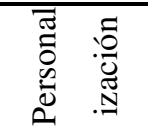 & Distingue públicos & $\begin{array}{l}\text { Público marginal (perfiles de poca } \\
\text { importancia para la organización) }\end{array}$ & 4 & $\begin{array}{r}\text { García Borbolla et al. } \\
\text { (2005); Castillo (2007), }\end{array}$ \\
\hline
\end{tabular}


La interactividasd en las sedes webs corporativas: retos y oportunidades para las pymes
Maria García; Ana Castillo Diaz;

M. Victoria Carrillo Durán

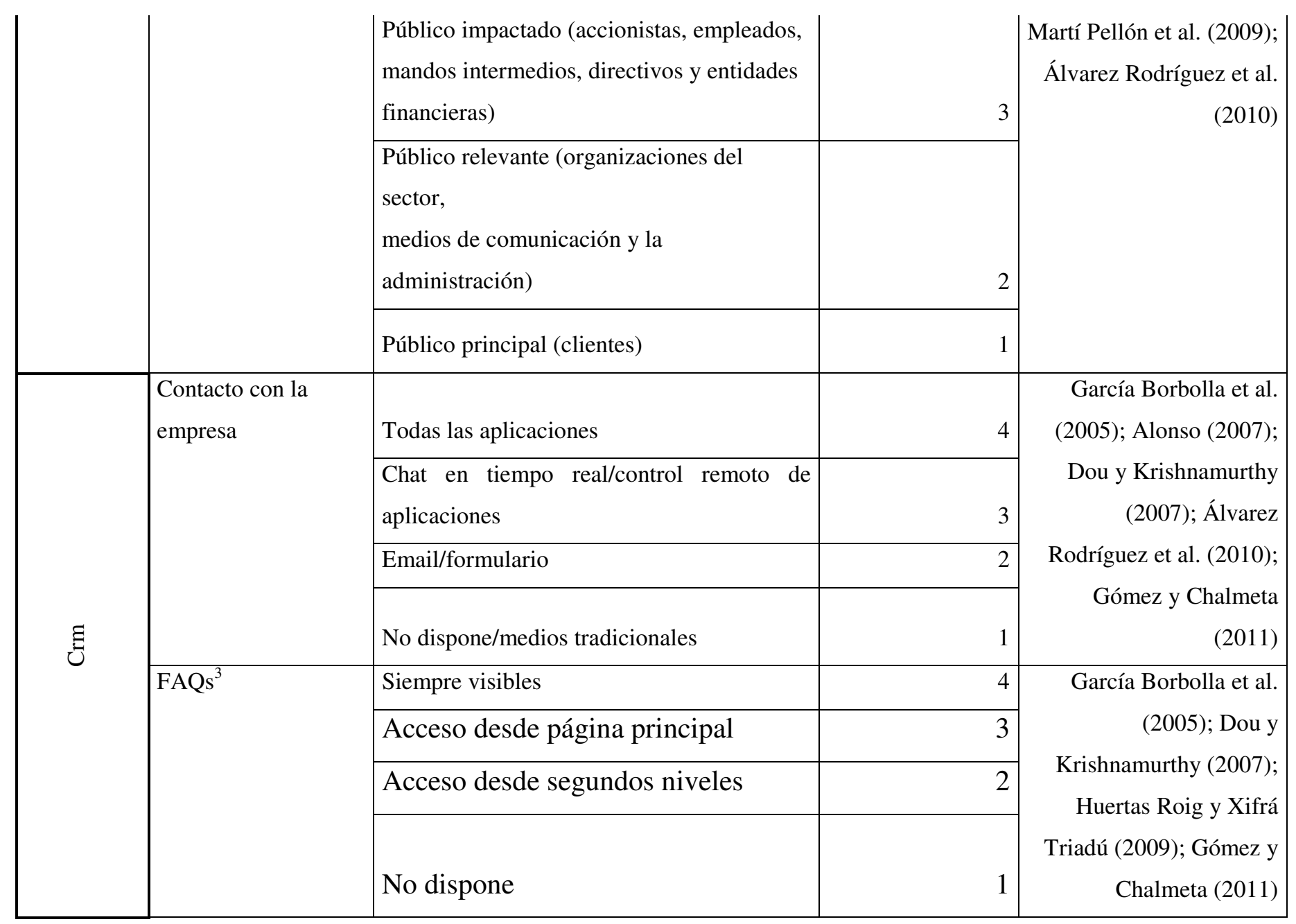

Fuente: Elaboración propia.

Figura 1 - La agrupación de factores obtenida tras el análisis factorial

\begin{tabular}{|c|l|}
\hline \multirow{2}{*}{ Factor 1 } & Suscripción \\
\cline { 2 - 2 } & Fomento de la comunidad \\
\cline { 2 - 2 } & Archivo de noticias \\
\cline { 2 - 2 } & Participación \\
\hline Factor 2 & FAQs \\
\cline { 2 - 2 } & Distingue públicos \\
\hline Factor 3 & Contacto \\
\hline
\end{tabular}

Fuente: Elaboración propia.

\footnotetext{
${ }^{3}$ Frequently asked questions, voz inglesa que en castellano se traduce por "preguntas frecuentes".
} 